\title{
Research status and hot spot visualization analysis of "gas safety" based on CiteSpace
}

\author{
Ru Li, Hui Huang*, Jia Zhang, Mengxin Zhang \\ School of Materials and Chemical Engineering, Ningbo University of Technology, Ningbo, Zhejiang, 315211, P R China
}

\begin{abstract}
Taking "gas" and "safety" as the theme, this paper adopts bibliometric method and visual analysis method, and makes use of CiteSpace software to analyze the current research situation in the field of domestic gas safety in terms of keywords, authors, institutions, etc. , understand the research process hot spots and risk points, in order to better promote gas safety management. The results show that at present, there are many hot spots in gas safety research, especially in safety management. Many excellent authoritative scholars published papers, some of the key authors have initially established a small-scale literature cooperation network and close contact. In contrast, the agency appears slightly single, the research force is more scattered. More universities should be encouraged to participate in research on related topics, and inter-agency cooperation and exchanges at a deeper level should be strengthened to share development results and gradually form stable cooperation clusters.
\end{abstract}

\section{Introduction}

With the continuous expansion of gas application market scale, China has entered the gas era, and is currently in the critical period of energy structure transformation and in-depth development. City gas has good potential value and can provide a good internal driving force[1] for energy structure transformation and further development. However, due to the characteristics of flammable, explosive and toxic gas, once the facilities leak, fire and poisoning accidents are easy to occur. Statistics show that in 2019 gas accident news a total of 722 , related casualties: 63 people were killed and 585 injured. In this context, gas safety research is particularly important, widely concerned by scholars. In recent years, gas safety related research has been extensive and hot research branches are numerous. At the same time, the diversity of research centers also provides a strong theoretical basis for safety management and security system. It is helpful to further improve the quality of gas safety management in China. CiteSpace software is used to visualize the gas safety field in 2010-2019 to understand the research hot spots, development trends and frontiers in this field.

\section{Consequence}

\subsection{Time distribution}

Figure 1 shows the number of thematic literature published in each year. In the past ten years, gas safety related research has been carried out continuously, and the volume of communications has generally shown a steady upward trend. In 2017 and 2019, it reached a peak state, with a maximum of 245 articles.

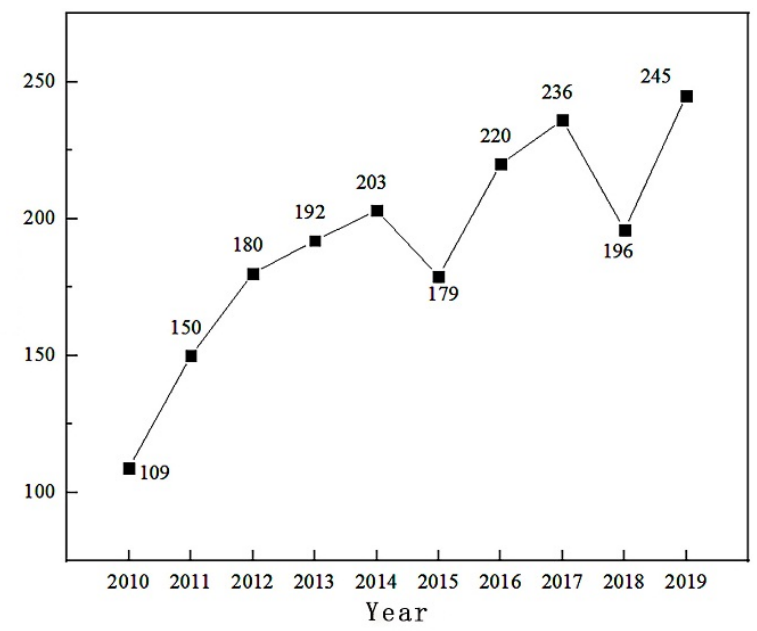

Figure 1. Gas safety literature published from 2010 to 2019.

\subsection{Analysis of key words}

CiteSpace provides a combination of keywords and terms, Can explore a certain field of common concern of scholars topic[2]. This means that the research focus or focus topic in a certain period can be reflected by high frequency keywords. In the circle link mark, according to its internal width and color, the frequency[3] of node content in each time stage can be represented. Figure 2 shows the results of keyword visualization analysis in 2010-2019, with 353 nodes, 510 connections, density of 0.0082 . Where the size of the node represents the frequency of keywords, Figure 2 shows gas safety (306

"Corresponding author's e-mail: huihuang@nbut.edu.cn 
times), safety management (227 times), gas (103 times), gas pipeline (103 times) and safety (100 times) are the keywords with higher frequency. Mediocentricity (or centrality) is one of the important indicators of the degree of connection between response nodes, darkness of the contour color represents the size[4] of the mediation centrality of the node. The minimum number of paths $s$ to the node $t$ through the centrality formula $\mathrm{BC}_{t}-\sum_{t+t+t} \frac{n_{s t}^{t}}{g_{s t}}$,

$\mathrm{n}_{\text {st }}^{\mathrm{i}}$ the number of shortest paths through the node $\mathrm{i}$ in $\mathrm{g}_{\mathrm{st}}$ shortest path from the node $s$ to the node), the node greater than 0.1 can be calculated as an important node[5]. Gas, gas pipeline, gas safety and so on as important nodes, with other nodes, the branch surface is more extensive. Meanwhile, gas safety is also the most frequent keyword, unified with the initially set theme words. Close to them are fire engines, fire brigades, Yanjiao towns, electrical lines and fire stations, a small network of knowledge is formed.

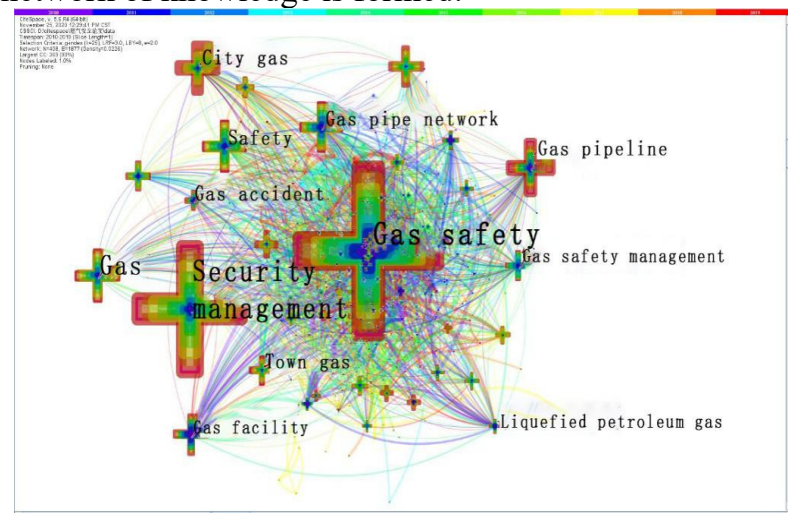

Figure 2. Keywords of gas safety co-occurrence diagram.

Any single perspective and analytical dimension is difficult to grasp the full picture of the scientific frontier, because the scientific frontier itself is in the process of dynamic and development, especially the trend of intersection and integration between disciplines is speeding up[6], abstract-based emergent word detection reflects the dynamic changes in the research field and helps to present some potential research frontiers. With the algorithm Kleinberg in the CiteSpace, figure 3 is obtained by intercepting the first 16 of the keyword outburst information table. The figure "Year" represents the year the data begins to collect," Strength" is the outburst rate, representing the outburst intensity of the keyword, and the city, gas facility and gas pipeline keywords are the highest. Cities, gas leakage, solutions, gas construction and the status of key words in 2015-2019, the emergence of the degree of sustained high, taken together, that scholars have shifted to these areas of focus and focus on this. This is in line with the national policy of speeding up the development of urban gas and energy conservation in recent years and the policy of safety and power. Because of the improvement of living standards of residents in China, as a new gas fuel, gas is recognized by the broad masses of the people for its convenience, but the subsequent safety problems are gradually aggravated. It is inferred that the focus of research in the next few years will gradually turn to urban gas.

Top 16 Keywords with the Strongest Citation Bursts

\begin{tabular}{lllll} 
Keywords & \multicolumn{5}{c}{ Year } & Strength Begin & End & $\mathbf{2 0 1 0}$ - 2019 \\
Tianjin & 2010 & 3.1071 & $\mathbf{2 0 1 0}$ & 2013 \\
Gas facility & 2010 & 3.5184 & $\mathbf{2 0 1 0}$ & 2012 \\
Safety technology & 2010 & 2.4009 & $\mathbf{2 0 1 0}$ & 2013 \\
City Gas Pipeline & 2010 & 2.1037 & $\mathbf{2 0 1 1}$ & 2014 \\
User security & 2010 & 1.9628 & $\mathbf{2 0 1 2}$ & 2014 \\
Analysis & 2010 & 2.1503 & $\mathbf{2 0 1 2}$ & 2015 \\
Management measures & 2010 & 2.9344 & $\mathbf{2 0 1 3}$ & 2015 \\
Quality & 2010 & 2.3127 & $\mathbf{2 0 1 3}$ & 2015 \\
Public safety & 2010 & 1.9265 & $\mathbf{2 0 1 3}$ & 2015 \\
Accident & 2010 & 2.2374 & $\mathbf{2 0 1 3}$ & 2015 \\
Gas pipeline & 2010 & 3.2705 & $\mathbf{2 0 1 4}$ & 2016 \\
City & 2010 & 5.1122 & $\mathbf{2 0 1 6}$ & 2019 \\
Gas 1eakage & 2010 & 2.7428 & $\mathbf{2 0 1 6}$ & 2019 \\
Solution & 2010 & 2.2831 & $\mathbf{2 0 1 6}$ & 2019 \\
Gas construction & 2010 & 2.6191 & $\mathbf{2 0 1 6}$ & 2019 \\
\hline
\end{tabular}

Figure 3. Highlight word map.

\subsection{Author analysis}

Through the study of the authors, we can grasp the breadth and depth of scientific research activities, which is of positive significance to the organization, management and coordination of scientific research activities. Figure 4 shows a graph of author co-occurrence with 424 nodes, 406 connections, and 0.0045 network density. The size of the nodes and the connections represent the authors, output and the relationship between them. As can be seen from Figure 4, the authors who have published a lot of articles are Shao Zehua (28 articles), Yan Guodong (21 articles), Zhang Tianming (21 articles), Gao Jie (21 articles), Guo Libin (21 articles) and so on. Shao Zehua is the general manager of Chengdu Qinchuan Internet of Things Technology Co., Ltd. His company's main business direction is the research and development of intelligent gas meters. In the past ten years, he has followed up the research in the field of gas safety and continued to output the results of his papers, a thorough understanding of the relevant knowledge is an important contribution to expanding the gas application market. According to Prices law, the formula $N_{\min }=0.749 \sqrt{N_{\max }}, N_{\min }$ is the minimum number of articles published by core authors and $N_{\min }$ is the highest number of articles published by authors. Through calculations, those with 4 articles or more are core authors[7], which are important forces to promote the development of gas safety related research. We are easily to find that each author has a relatively high amount of articles, showing a dot-like distribution. At the same time, by analyzing the network density, it can be found that the cooperation intensity between authors is not high. High-frequency authors such as Gao Jie, Guo Libin, and Peng Zaimei are basically independent researches. Therefore, the mutual cooperation between authors needs to be improved, and 
the cooperation is formed in the form of point-to-face research. Network can strengthen research in the field of gas safety.

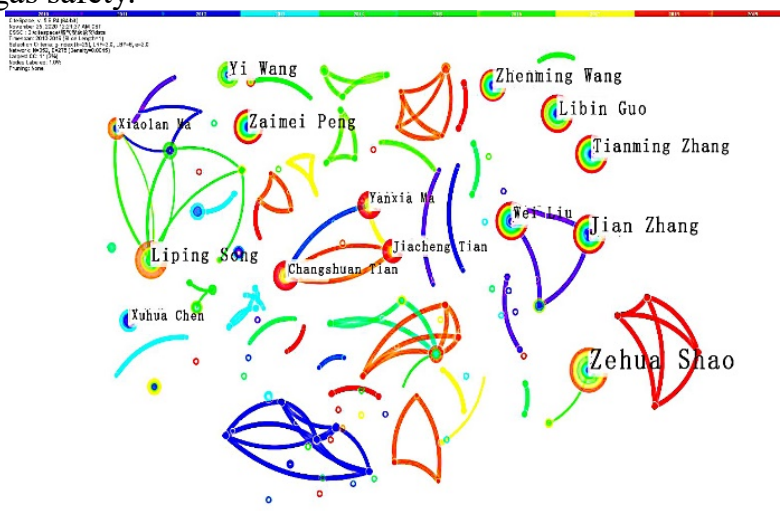

Figure 4. Co-appearance of authors.

\subsection{Analysis of publishing institutions}

As a new perspective to evaluate the academic influence of scientific researchers, countries or institutions, the cooperation map intuitively shows the social relations between scholars in a certain research field, countries or research institutions[8]. After Node types are set to institution to run the software, the co-occurrence diagram of research institutions is obtained, as shown in Figure 5. The nodes in the figure correspond to the institutions one by one, and each color value on the time axis determines a unique year, which corresponds to the color of the node outline in the cooperative co-occurrence network. Combined with the analysis of Table 3 , it is found that the research network branch covered by Beijing Gas Group Co., Ltd. and Beijing University of Civil Engineering and Architecture has a wide and dense coverage, which indirectly promotes Tianjin Gas Group Co., Ltd., Shaanxi Datang Intelligent Instrument Co., Ltd. and Beijing Municipal Engineering. The academic exchange and communication of the Central Design Research Institute Co., Ltd. Based on the analysis of the number of publications, the institutions with the most publications are Chengdu Qinchuan Internet of Things Technology Co., Ltd. (26 articles), China Municipal Engineering North China Design and Research Institute (22 articles), and PetroChina Kunlun Gas Co., Ltd. Shandong Branch ( 22 articles), Wuxi City Gas Management Office (21 articles), Golmud China Resources Gas Co., Ltd. (21 articles), etc. According to analysis, most of the above institutions belong to independent research status and have not tended to form cooperative groups. However, the number of published literatures is outstanding, which is still the backbone of gas safety related research.

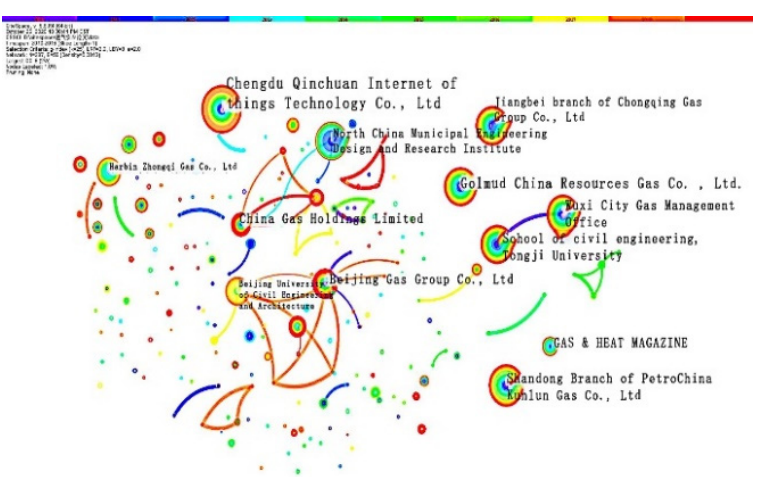

Figure 5. The co-appearance network of publishing institutions.

\section{Discussion of results}

This article takes gas safety as the theme, and 1913 related documents in the CNKI database from 2010 to 2019 as the research object. The CiteSpace visualization software is used to draw a scientific knowledge map. According to the research status, it is based on the three perspectives of keywords, publishing authors and publishing organizations. After an in-depth analysis, the following conclusions are drawn:

(1) The top three keywords in frequency are gas safety, safety management and gas, and risk management and safety technology are the main emergent words. With the vigorous development of the gas industry, the importance of gas safety management has become increasingly prominent. We can strengthen the gas safety management, reduce the probability of gas accidents and reduce the loss caused by accidents by strengthening the attention, improving the safety management system, comprehensively improving the safety skills of staff and expanding the popularization scope of safety awareness.

(2) From the analysis of the graph by the author, it can be seen that the number of articles published by a single author is relatively high, which has greatly promoted the development of the field of gas safety. Generally speaking, the cooperation between the authors is relatively lacking. High-volume authors such as Gao Jie, Guo Libin, and Peng Zaimei are all independent researchers. The authors can communicate through related academic exchanges in the gas field to promote joint cooperation and strengthen gas safety. Field of research.

(3) From the perspective of document issuing institutions, colleges and universities and local listed gas companies are the mainstay of the research in this field. Chengdu Qinchuan Internet of things Technology Co., Ltd., North China Municipal Engineering Design and Research Institute, and Shandong Branch of PetroChina Kunlun Gas Co., Ltd. have issued outstanding papers, which are important components. Based 
on the whole, the organization has not formed a certain scale of cooperation and exchange system. Beijing Gas Group Co., Ltd. and Beijing Jianzhu University are the key hubs for promoting research cooperation between domestic gas safety related institutions in various provinces. Their concentrated cooperation promotes academic exchanges and common development. It is worth learning from other institutions.

\section{Acknowledgments}

This work was supported by Student Innovation and Entrepreneurship Training Program of China (202011058005) and the Student Research Program of Ningbo University of Technology (2020034) and Ningbo Education Science Planning Project (2019YZD010).

\section{References}

1. Chen S.N. (2019) Current Situation and Countermeasures of Urban Gas Safety Management under the New Situation. Housing and Real Estate, 36: 133.

2. Li, J., Chen, C.M. (2016) CiteSpace Technology Text Mining and Visualization (2nd Edition).
Capital University of Economics and Business Press, Bei Jing.

3. Wu, QY., Zheng, L., Su, X.Y., Xu, H.L. (2019) Visualization analysis of chemical safety based on Citespace. Edu. Modern., 6: 229-232.

4. Ling, X., Zhao, Z.G., Li, X.G. (2019) Application of artificial intelligence technology in pharmaceutical field: visualization analysis of literature based on Web of Science. Chin. Pharm., 30: 433-438.

5. Li, J., Chen, C.M. (2017) CiteSpace Technology Text Mining and Visualization (2nd Edition). Capital University of Economics and Business Press. Bei Jing.

6. Harold A L. (2011) Three Eras of Technology Foresight. Technovation,31:69-76.

7. Ye, G., Fu, Y., Wang, Y.H. (2019) Review of research on building safety based on CiteSpace from the perspective of behavior. Saf. Environ. Eng., 26: 127-134.

8. Chen, Y., Chen, C.M., Liu, Z.Y. (2015) Methodological function of CiteSpace knowledge Atlas. Stud. Sci. Sci., 33: 242-253. 